“ (C) 2018 IEEE. Personal use of this material is permitted. Permission from IEEE must be obtained for all other uses, in any current or future media, including

reprinting/republishing this material for advertising or promotional purposes, creating new collective works, for resale or redistribution to servers or lists, or reuse of any copyrighted component of this work in other works." 


\title{
Particle Swarm Optimized, 3D-Printed, Wideband, Compact Hemispherical Antenna
}

\author{
Ming-Chun Tang, Senior Member, IEEE, Xiaoming Chen, Student Member, IEEE, Mei Li, Member, \\ IEEE, and Richard W. Ziolkowski, Fellow, IEEE
}

\begin{abstract}
A 3D-printed, wideband, compact hemisphericalshaped antenna is presented. It consists of a driven strip monopole and several parallel near-field resonant parasitic (NFRP) strips that reside on the surfaces of a hemispherical shell. The monopole strip lies on the interior surface; the NFRP strips lie on the exterior one. This arrangement facilitates the requisite stable near-field capacitive coupling between them over a wide frequency range. The particle-swarm optimization (PSO) algorithm is used to define the lengths and locations of these NFRP strips to achieve its optimized operational bandwidth around $700 \mathrm{MHz}$ given its compact size. The hemispherical shell was 3D printed with acrylonitrile butadiene styrene (ABS); the strips were applied to it with silver paste. This prototype was tested. The measured results, in agreement with their simulated values, demonstrate that it achieves a $17.97 \%-10-\mathrm{dB}$ fractional impedance bandwidth over which stable realized gain values, near $3.5 \mathrm{dBi}$, are attained. With its low-cost fabrication and attractive performance characteristics, this $3 \mathrm{D}$ printed antenna is suitable for indoor multi-path wireless communication systems.
\end{abstract}

Index Terms-3D printing, compact antenna, near-field resonant parasitic (NFRP) elements, PSO algorithm, spherical antenna, wide bandwidth

\section{INTRODUCTION}

$\mathrm{C}$ ompact antennas commonly suffer from low bandwidth and overall efficiency. These drawbacks limit their widespread applications in wireless communication systems [1-3]. Several designs have been developed recently to explore the impedance bandwidth limits. These include spherical and semi-spherical wire arrangements [4-5], wire cage structures [6], and the utilization of the multiple near-field resonant parasitic (NFRP) elements [7-9].

Manuscript received on Mar. 30, 2018, revised on May 26, 2018

This work was supported in part by the National Natural Science Foundation of China contract number 61471072, in part by the Funding of the Innovative Leading Talents in Science and Technology of Chongqing contract number CSTCCXLJRC201705, in part by Funding of the Young Backbone Teachers in Colleges and Universities of Chongqing contract number 0307001104102, in part by the Opening Subject of State Key Laboratory of Millimeter Waves under Contract K201732, and in part by the Australian Research Council grant number DP160102219.

M. -C. Tang, X. Chen, and M. Li are with the Key Laboratory of Dependable Service Computing in Cyber Physical Society Ministry of Education, College of Communication Engineering, Chongqing University, Chongqing 400044, China, and also with the State Key Laboratory of Millimeter Waves, Nanjing 210096, China (e-mail: tangmingchun@cqu.edu.cn);

R. W. Ziolkowski is with the University of Technology Sydney, Global Big Data Technologies Centre, Ultimo NSW 2007, Australia, and the Department of Electrical and Computer Engineering, University of Arizona, Tucson, AZ 85721, USA (e-mail: richard.ziolkowski@uts.edu.au).
Compared with standard optimization techniques, intelligent algorithms have been employed to design antennas due to their superior efficacy in achieving desired results [10-14]. The particle swarm optimization (PSO) algorithm is a proven example $[12,14]$. The basic PSO approach has premature convergence issues and difficulties when processing multi-object or high-dimensional problems. Recently, adapted PSO algorithms have been shown to have an improved ability to search for optimal solutions $[12,14]$.

Additive manufacturing (AM), often termed 3D printing, has attracted significant attention recently from a wide variety of research and commercial entities. It is able to produce arbitrarily shaped components without high-cost moldings and complex procedures. Successful 3D printing of a variety of microwave devices has confirmed the feasibility and merits of this technology. These examples include radio-frequency waveguides [15], filters [16], circuits [17], and antennas [15, 18-20]. Furthermore, 3D printing offers a precision of $0.05 \mathrm{~mm}$, which is acceptable to accurately fabricate a variety of small-sized antenna designs.

In this letter, a 3D-printed, wideband, compact, hemispherical-shaped, near-field resonant parasitic antenna is reported. Several parallel metallic strips act as NFRP elements that are oriented orthogonal to a driven monopole strip. They are embedded in the surfaces of its 3D-printed hemispherical shell, which rests on a copper ground plane. It is demonstrated that the presence of these sub-wavelength sized NFRP strips introduces additional resonances that lead to the enhanced bandwidth. With the aid of the adapted PSO algorithm, the dimensions and locations of all of these strips are optimized to produce a wide impedance bandwidth while maintaining high overall efficiency. A prototype operating around $700 \mathrm{MHz}$ was fabricated. The hemispherical shell was 3D printed with acrylonitrile butadiene styrene (ABS). Conducting silver paste realized the NFRP and driven monopole strips embedded into it. This prototype was tested and the measured results, in agreement with their simulated values, indicate that the reported antenna is compact. The hemispherical radiator is $k a_{\text {radiator }} \sim 0.835$ in size, where $a_{\text {radiator }}$ is the outer radius of the hemisphere and $k=2 \pi / \lambda_{L}=2 \pi f_{L} / \mathrm{c}$ is the corresponding free space wave number at $f_{L}$, the lowest frequency within its operational band. It achieves a $17.97 \%-10-\mathrm{dB}$ fractional impedance bandwidth and produces stable realized gain values near $3.5 \mathrm{dBi}$ within this operational bandwidth. Note, however, that the cross-polarization levels of this antenna are quite high, which is different from previously reported wideband NFRP antennas [7-9]. On the other hand, this feature actually enables it to be a good candidate for potential applications in indoor 
multi-path wireless communication systems [21-23].

\section{ANTENNA DESIGN}

The basic configuration is depicted in Fig. 1. A hemispherical shell is $3 \mathrm{D}$ printed with the commonly used material, ABS resin, whose relative dielectric constant is $\varepsilon_{r} \sim$ 3.01 . The shell is printed with grooves having a $0.2 \mathrm{~mm}$ depth where the conductors will be placed [24]. The 3D-printing machine, ZRapid SLA500, was employed and driven with the software, Magics. The substructure was specified in STL format and was layer-by-layer printed using stereo lithography appearance (SLA) technology. Quick-dry silver paste filled its grooves. The outer radius of the shell is $59.85 \mathrm{~mm}$ and its thickness is $2.0 \mathrm{~mm}$. In order to ensure the desired antenna performance, the dried silver paste had to be thick enough and reasonably flat to perform as continuous metal strips without any discontinuities.

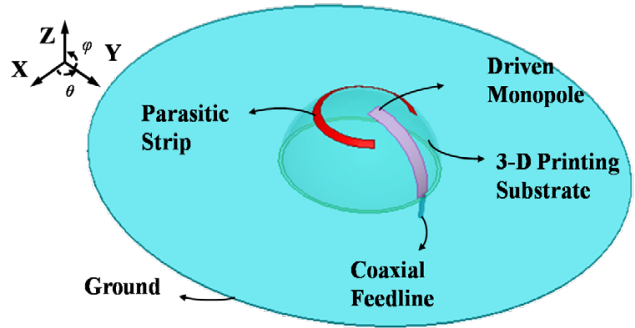

Fig. 1 The basic configuration of the 3D-printed NFRP antenna.

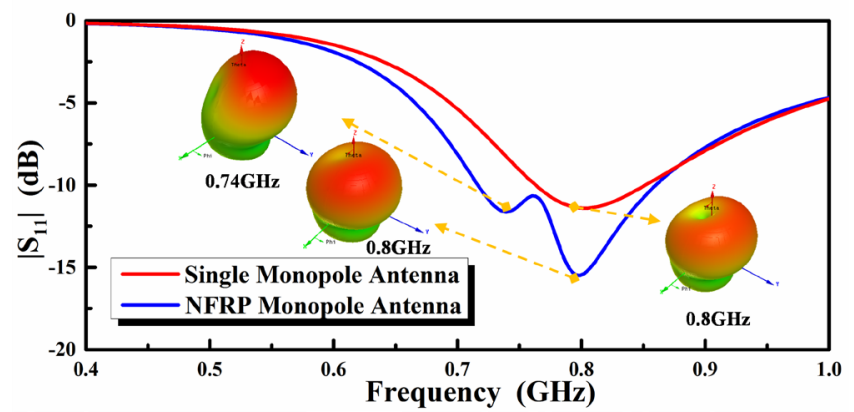

Fig. 2 Simulated reflection coefficient values of the 3D-printed NFRP antenna and the monopole antenna alone as functions of the source frequency. Insets are the $3 \mathrm{D}$ realized gain patterns at the indicated frequencies.

The radiating elements of the antenna consist of a driven monopole and a circular capacitively loaded loop (CLL) parasitic strip. The driven monopole is etched on the inside surface of the shell. It is directly connected to the inner conductor of the coaxial feedline which passes through a hole in the ground plane. The outer conductor of the coax is soldered to the circular copper ground disc whose radius is $160 \mathrm{~mm}$. Since the driven monopole is conformal to the hemisphere, its height is lower than a standard electric monopole operating at the same frequency point. The electrical size of the hemispherical radiator with only the monopole present is $k a_{\text {radiator }}=0.955$. As shown in Fig. 2 , its fractional bandwidth is $10 \%$. Moreover, its radiation pattern is quite different from a standard electric monopole. The null is not as deep and is tilted away from the broadside direction, i.e., away from the +z-axis. The CLL strip is oriented parallel to the ground plane and orthogonal to the monopole. It is embedded in the outside surface of the shell and serves as the NFRP element of the antenna [25]. Its resonance frequency is determined to be close to, and below that of the monopole. Combined with the monopole, the overall fractional bandwidth is improved to $15 \%$. Moreover, because the lower end of the impedance bandwidth is now lower, it has a smaller electrical size, $k a_{\text {radiator }}=0.898$. Note that, as will discussed below, the arrangement of the driven and NPRP elements leads to cross polarization levels in the radiation patterns that are much higher than a standard monopole over the entire operation band.

As shown in Figs. 3(a) and (c), more parallel NFRP CLL strips are added to the outer surface of the shell with a uniform gap, $\varphi_{I}=6.5^{\circ}$, to further expand the bandwidth. The widths of all of the NFRP strips were optimized to occupy the same arc: $\varphi_{2}=6^{\circ}$. The angular offset of these NFRP CLL elements in a vertical cut and the widths of their gaps are quantified with two angular units denoted as $\theta_{1}-\theta_{5}$ and $\theta_{6}-\theta_{10}$, respectively, in Fig. $3(\mathrm{~b})$. The length of the monopole is specified by the angle $\varphi_{3}=$ $83^{\circ}$ from horizontal and its width is $13.0 \mathrm{~mm}$.

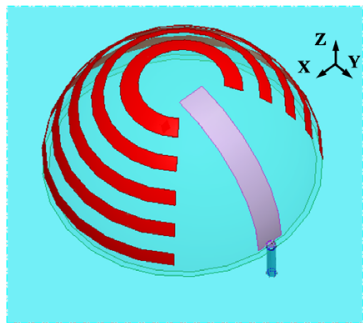

(a)

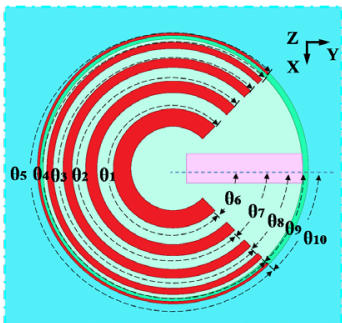

(b)

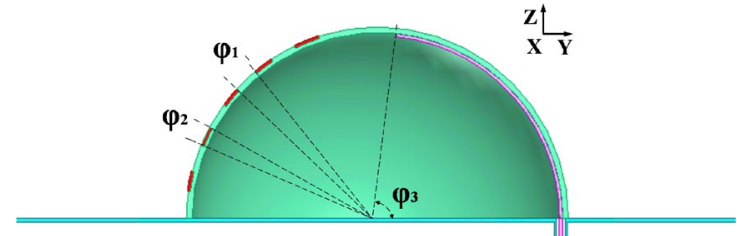

(c)

Fig. 3 The adapted PSO defined 3D-printed NFRP antenna. (a) 3D view. Top (b) and side (c) views of the hemispherical radiating structure.

An adapted PSO algorithm is used to define the optimized values of $\theta_{1}$ to $\theta_{10}$. Each set of these parameters is represented by a 10-dimensional position vector $\boldsymbol{X}$ of a particle. For each iteration $k$, the particle's position $\boldsymbol{X}$ and velocity $\boldsymbol{V}$ are changed according to the previous velocity $\boldsymbol{V}$ and position $\boldsymbol{X}$ with the following update equations:

$$
\begin{aligned}
& \boldsymbol{V}_{i}^{k+1}=w_{i}^{k} \boldsymbol{V}_{i}^{k}+c_{1} r_{I}\left(X_{i}^{\text {Best }}-\boldsymbol{X}_{i}^{k}\right)+c_{2} r_{2}\left(X_{\text {all }}^{\text {Best }}-\boldsymbol{X}_{i}^{k}\right) \\
& \boldsymbol{X}_{i}^{k+1}=\boldsymbol{X}_{i}^{k}+\boldsymbol{V}_{i}^{k}
\end{aligned}
$$

The inertia weight $w$ is adapted according to the fitness value of each particle to avoid premature convergence [26-27]. The detailed inertia weight transformations are specified by the following system of iteration update equations:

$$
\begin{gathered}
w_{i}^{k}=1-\frac{1-e^{-k}}{1+e^{-k}}+w_{\min } \quad \text { if Fitness }{ }_{i}^{k}<\text { Fitness }_{\text {avg }}^{k} \\
w_{i}^{k}=w_{\max }-\left(w_{\max }-w_{\min }\right)\left(1-e^{\frac{-k}{k \max }}\right) \text { if Fitness }{ }_{i}^{k}<\text { Fitness }_{\text {havg }}^{k}
\end{gathered}
$$




$$
w_{i}^{k}=w_{\max }-w_{\min } \frac{\text { Fitness }_{\text {havg }}^{k}-\text { Fitness }_{i}^{k}}{\mid \text { Fitness }_{\text {max }}^{k}-\text { Fitness }_{\text {havg }}^{k} \mid} \text { if Fitness }{ }_{\text {havg }}^{k}<\text { Fitness }_{i}^{k}
$$

where Fitness ${ }_{\text {max }}^{k}$ represents the maximum fitness value among all the particles; Fitness savg represents the average fitness value among all the particles; and Fitness $s_{\text {havg }}^{k}$ represents the average fitness value among the particles whose fitness value is larger than Fitnessavg ${ }_{\text {arg }}^{k}$

To get the desired wide impedance bandwidth in this algorithm, the fitness value function is set by accumulating the reflection coefficients after multiple trails as:

$$
\text { Fitness }_{i}^{k}=f\left(\boldsymbol{X}_{i}^{k}\right)=\sum\left|\mathrm{S}_{11}\right| \mathrm{dB}
$$

where $f\left(\boldsymbol{X}_{i}^{k}\right)$ is the accumulated value of the reflection coefficients of 41 frequency points from 600 to $800 \mathrm{MHz}$ with a step of $5 \mathrm{MHz}$. Note that if the $\left|\mathrm{S}_{11}\right|$ value of one frequency point is below $-10 \mathrm{~dB}$, only -10 is added into Eq. (6). The initial value of each design parameter is set randomly in the range listed in Table I. The other configurations of the adapted PSO algorithm are listed in Table II.

TABle I. The Ranges of the Initial Design Parameters of the ANTenNa (UNITS: DEGREES $\left({ }^{\circ}\right)$ )

\begin{tabular}{|c|c|c|c|}
\hline Parameters & Range & Parameters & Range \\
\hline$\theta_{1}$ & {$[180,330]$} & $\theta_{2}$ & {$[180,330]$} \\
\hline$\theta_{3}$ & {$[180,330]$} & $\theta_{4}$ & {$[180,330]$} \\
\hline$\theta_{5}$ & {$[180,330]$} & $\theta_{6}$ & {$[0,360]$} \\
\hline$\theta_{7}$ & {$[0,360]$} & $\theta_{8}$ & {$[0,360]$} \\
\hline$\theta_{9}$ & {$[0,360]$} & $\theta_{10}$ & {$[0,360]$} \\
\hline
\end{tabular}

TABle II. The Configurations Of The PSO Algorithm

\begin{tabular}{|c|c|c|c|}
\hline \multicolumn{2}{|c|}{ Number of Particles: $i$} & \multicolumn{2}{c|}{30} \\
\hline \multicolumn{2}{|c|}{ Number of Iterations: $k_{\max }$} & \multicolumn{2}{c|}{60} \\
\hline$c_{1}$ & 1.8 & $c_{2}$ & 1.8 \\
\hline$r_{1}$ & $\operatorname{rand}(0,1)$ & $r_{2}$ & $\operatorname{rand}(0,1)$ \\
\hline$w_{\max }$ & 0.8 & $w_{\min }$ & 0.2 \\
\hline
\end{tabular}

The co-simulation is carried out by running the PSO algorithm and HFSS together. The individual steps used for our adapted PSO solution are:

Step 1: Set iteration $k=1$ and initialize the position $\boldsymbol{X}$ and velocity $\boldsymbol{V}$ of all particles.

Step 2: Evaluate fitness value of each particle according to Eq.(6). Stop the procedure when the iteration $k$ is up to $k_{\max }$. Otherwise, proceed to Step 3.

Step 3: Analyze the fitness value of each particle. Update the group's best position and each particle's best position. Each particle's inertia weight $w$ is then calculated from its previous fitness value according to Eqs. (3)-(5).

Step 4: Update each particle's velocity and position with Eqs. (1) and (2).

Step 5: Set iteration to $k+1$ and return to Step 2.

Then Matlab generates a VB scripts file and launches HFSS to run a simulation with the specified design parameters. After completion, the reflection coefficients are passed from HFSS back to the adapted PSO algorithm in Matlab and another iteration begins. The entire optimization process takes up to 72 hours. Note that most of this time is occupied by the HFSS simulations. In contrast, the PSO algorithm running in Matlab requires little time.

The selected fitness function led to an efficient optimization process. The adapted PSO algorithm basically converged between the $40^{\text {th }}$ and $50^{\text {th }}$ iteration for most of our trials. Fig. 4 shows the best convergence result of the fitness values we obtained. It is seen that the algorithm attained the global optimal design after the $49^{\text {th }}$ iteration. When it terminated, it had decreased to an acceptable value, -275 . The finalized design parameters, $\theta_{I}$ to $\theta_{10}$, are listed in Table III.

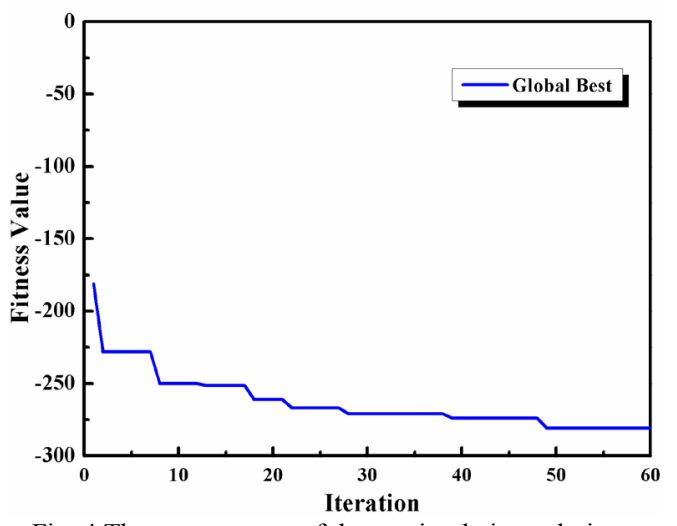

Fig. 4 The convergence of the co-simulation solution.

TABle III. The Final Wideband ANTENNA Design PARAmeters (UNITS: DEGREE $\left(^{\circ}\right)$ )

\begin{tabular}{|c|c|c|c|}
\hline$\theta_{1}=282$ & $\theta_{2}=258$ & $\theta_{3}=242$ & $\theta_{4}=182$ \\
\hline$\theta_{5}=188$ & $\theta_{6}=233$ & $\theta_{7}=232$ & $\theta_{8}=241.5$ \\
\hline$\theta_{9}=199$ & $\theta_{10}=132$ & \multicolumn{2}{|c|}{ NULL } \\
\hline
\end{tabular}

\section{Measured And Simulated Results}

The prototype of the optimized antenna was fabricated and assembled; a photo of it is given in Fig. 5(a). We note that during the assembly process the driven element is constructed by using copper foil tape instead of the silver paste for easy installation and a good connection to the inner conductor of the SMA.

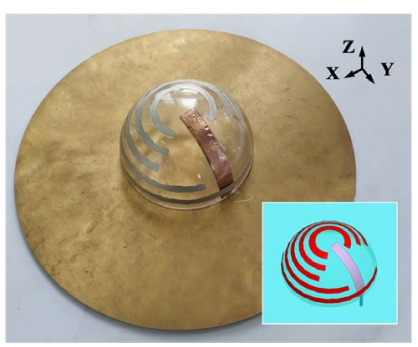

(a)

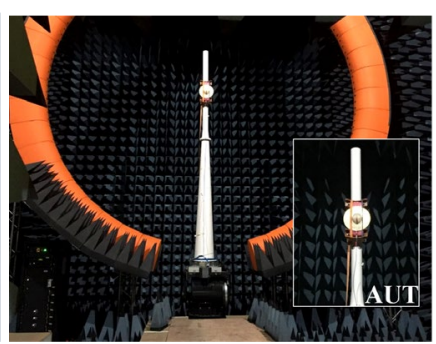

(b)
Fig. 5 The fabricated antenna. (a) Side view of the antenna after assembly. (b) Prototype antenna as the AUT in the measurement chamber.

The measured and simulation reflection coefficient values as functions of the excitation frequency are presented in Fig. 6(a). The measured results indicate that the antenna exhibits a wide -10-dB impedance bandwidth from 666 to $797.5 \mathrm{MHz}$ (131.5 $\mathrm{MHz}, \sim 17.97 \%$ fractional bandwidth); while the simulated 
values are from 689 to $816 \mathrm{MHz}(127.0 \mathrm{MHz}, \sim 16.87 \%$ fractional bandwidth). From the simulated values of the reference monopole alone, it is clear that the presence of the NFRP CLL elements introduced several overlapping resonant modes, which in turn produced the wide impedance bandwidth. Compared with the conformal antenna designs reported in [18] and [28], our prototype has the advantage of realizing a much wider bandwidth at the cost of a slightly larger electrical size.

The far-field radiation measurements were carried out in the multi-probe spherical near-field test anechoic chamber shown in Fig. 5(b) at the China Academy of Information and Communications Technology. The measured and simulated realized gain values at boresight as functions of the source frequency are presented in Fig. 6(b). It is readily seen that the measured (simulated) peak realized gain values are stable $\sim 3.5$ $\mathrm{dBi}(\sim 5 \mathrm{dBi})$ within the entire bandwidth. The simulated radiation efficiency is greater than $85 \%$ over the same bandwidth.

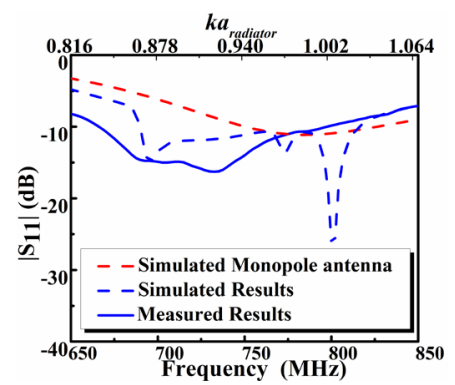

(a)

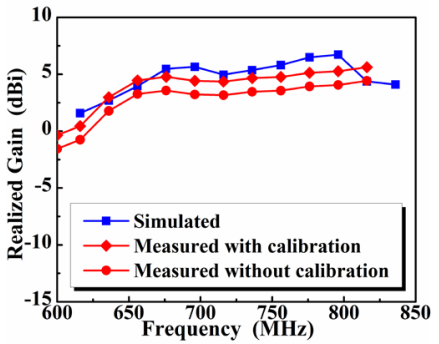

(b)
Fig. 6 Measured and simulated performance characteristics of the 3D printed antenna. (a) $\left|S_{11}\right|$ values as functions of the source frequency. The reference monopole values are also shown for comparison. (b) Realized gain as functions of the source frequency.

The measured (simulated) radiation patterns are given in Fig. 7 at arbitrarily selected frequency points in the lower: $696 \mathrm{MHz}$ (720 MHz), middle: $736 \mathrm{MHz}(756 \mathrm{MHz})$ and upper: $756 \mathrm{MHz}$ $(768 \mathrm{MHz})$ portions of the bandwidth. It is observed that the antenna produces relatively stable radiation patterns. It is also clear that the cross-polarization levels are much higher than those of a standard monopole. This characteristic is appealing for some special applications, notably for indoor wireless communications since it leads to better transmission capability in rich multipath environments [21-23].

On the whole, the measured results are in reasonable agreement with their simulated values. The electrical size of the hemispherical radiator in measurement (simulation) is 0.835 (0.864). The measured operational frequencies witnessed only a $3.3 \%$ red shift $(23 \mathrm{MHz})$ from their simulated values. The ground size affects the antenna performances, mainly the radiation pattern. According to our simulation studies, the antenna performance remains unchanged once the ground is large enough, (i.e., $\geq 160 \mathrm{~mm}$ ).

As confirmed by our simulation studies, the loss of the ABS resin substrate has little effect on the antenna radiation performances in the UHF band. Nonetheless, there are several main reasons for the clear differences between the simulated and measured results in Figs. 6 and 7. First, the silver paste was applied manually in our laboratory. Thus the precision of the strip widths was not very high. Moreover, the paste was not as conductive as expected. These factors were the main contributors to the redshift in the operational bandwidth seen in Fig. 6(a). Second, a $\sim 3 \mathrm{~m}$ long soft coaxial cable had to be used in the chamber. This inescapably introduced large transmission losses in the measured results $(\sim 1.2 \mathrm{~dB})$ and was responsible for the lower realized gain values seen in Fig. 6(b). Note that the calibrated realized gain values have also been presented in Fig. 6(b) for easy reference. Third, the tolerance errors in the 3D printing could not be avoided. They led to changes in the dimensions and shapes of the strips and the hemisphere, which also contributed to all of these noted differences.

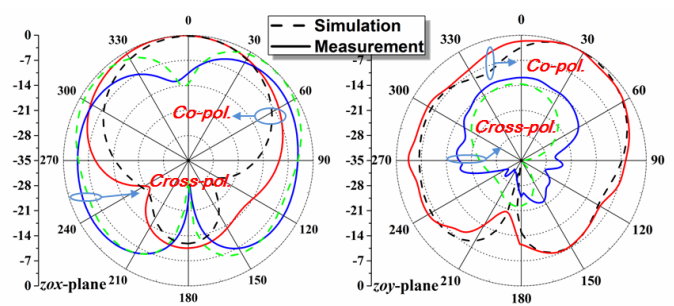

(a)

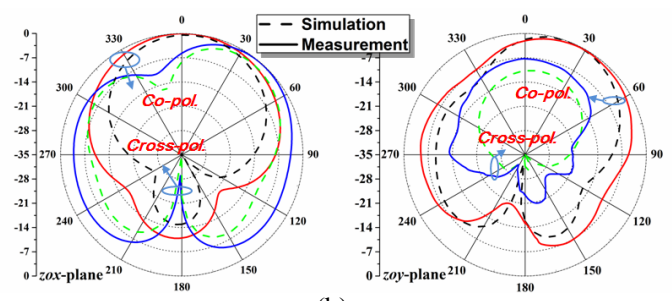

(b)

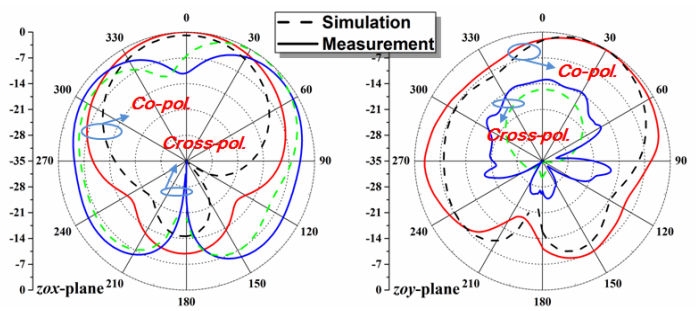

(c)

Fig. 7 Measured (simulated) normalized radiation pattern of the proposed antenna at selected frequency points; (a) at lower resonant point $696 \mathrm{MHz}$ (720 $\mathrm{MHz}$ ), (b) at middle resonant point $736 \mathrm{MHz}(756 \mathrm{MHz})$, and (c) at higher resonant point $756 \mathrm{MHz}(768 \mathrm{MHz})$.

\section{CONCLUSIONS}

A compact wideband hemispherical antenna was designed using an adapted PSO algorithm and realized with 3D printing. The resonances of its NFRP CLL elements broadened the impedance bandwidth of its driven monopole antenna. A 700 $\mathrm{MHz}$ prototype was fabricated and tested. The measured results demonstrated that the prototype achieved a $17.97 \%$ impedance bandwidth and attained realized gain values $\sim 3.5 \mathrm{dBi}$ with stable radiation patterns over it. Since it has improved broadside radiation and increased cross-polarization levels in comparison to the monopole alone, this NFRP antenna has potential for applications to indoor multi-path wireless communications. Since the fabrication and installation process is easily repeated without much effort, we anticipate that our antenna design can be scaled for mass production once the technology for processing metal components is included in future $3 \mathrm{D}$ printing machines or other conformal printing machines. 


\section{REFERENCES}

[1] L. J. Chu, "Physical limitations of omnidirectional antennas," J. Appl. Phys., vol. 19, pp. 1163-1175, 1948.

[2] J. S. McLean, "A re-examination of the fundamental limits on the radiation Q of electrically small antennas," IEEE Trans. Antennas Propag., vol. 44, no. 5, pp. 672-676, 1996.

[3] R.W. Ziolkowski and A. Erentok, "At and below the Chu limit: passive and active broad bandwidth metamaterial-based electrically small antennas," IET Microw. Antennas Propag., vol. 1, iss. 1, pp. 116-128, 2007.

[4] S. R. Best, "Electrically small resonant planar antennas: optimizing the quality factor and bandwidth," IEEE Antennas Propag. Mag., vol. 57, no. 3, pp. 38-47, Jun. 2015.

[5] O. S. Kim, "Low-Q electrically small spherical magnetic dipole antennas," IEEE Trans. Antennas Propag., vol. 58, no. 7, pp. 2210-2217, Jul. 2010.

[6] K. N. Madsen, Y. Zhou, and D. F. Sievenpiper, "A simplified low-Q electrically small magnetic dipole antenna," IEEE Antennas Wireless Propag. Lett., vol. 15, pp. 1975-1978, 2016.

[7] P. Jin, C.-C. Lin, and R.W. Ziolkowski, "Multifunctional, electrically small, planar near-field resonant parasitic antennas," IEEE Antennas Wireless Propag. Lett., vol. 11, pp. 200-204, 2012.

[8] M.-C. Tang, and R. W. Ziolkowski, "Two-element Egyptian axe dipole arrays emphasising their wideband and end-fire radiation performance," IET Microw. Antennas Propag., vol. 9, iss. 13, pp. 1363-1370, 2015.

[9] M.-C. Tang, R. W. Ziolkowski, S. Xiao, and M. Li, "A high-directivity, wideband, efficient, electrically small antenna system," IEEE Trans. Antennas Propag., vol. 62, no. 12, pp. 6541-6547, Dec. 2014.

[10] Y. Kuwahara, "Multiobjective optimization design of Yagi-Uda antenna," IEEE Trans. Antennas Propag., vol. 53, no. 6, pp. 1984-1992, Jun. 2005.

[11] A. Erentok and O. Sigmund, "Topology optimization of sub-wavelength antennas," IEEE Trans. Antennas Propag., vol. 59, no. 1, pp. 58-69, Jan. 2011.

[12] Y.-L. Li, W. Shao, L. You, and B.-Z. Wang, "An improved PSO algorithm and its application to UWB antenna design," IEEE Antennas Wireless Propag. Lett., vol. 12, pp. 1236-1239, 2013.

[13] E. Hassan, E. Wadbro, and M. Berggren, "Topology optimization of metallic antennas", IEEE Trans. Antennas Propag., vol. 62, no. 5, pp. 2488-2500, May 2014.

[14] A. Lalbakhsh, M. U. Afzal, and K. P. Esselle, "Multiobjective particle swarm optimization to design a time-delay equalizer metasurface for an electromagnetic band-gap resonator antenna," IEEE Antennas Wireless Propag. Lett., vol.16, pp. 912-915, 2017.

[15] A. I. Dimitriadis, T. Debogovic, M. Favre, M. Billod, L. Barloggio, J. Ansermet, and E.Rijijk, "Polymer-based additive manufacturing of high-performance waveguide and antenna components," Proc. IEEE, vol. 105, no. 4, pp. 668-676, Apr. 2017.

[16] B. Zhang, and H. Zirath, "3D printed iris bandpass filters for millimetre-wave applications," Electron. Lett., vol. 51, no. 22, pp. 1791-1793, Oct. 2015.

[17] J. A. Byford, M. I. M. Ghazali, S. Karuppuswami, B. L. Wright, and P. Chahal, "Demonstration of RF and microwave passive circuits through 3D printing and selective metalization," IEEE Trans. Compon. Packag. Manuf. Technol., vol. 7, no. 3, pp. 463-471, Mar. 2017.

[18] J. J. Adams, E. B. Duoss, T. F. Malkowski, M. J. Motala, B. Y. Ahn, R. G. Nuzzo, J. T. Bernhard, and J. A. Lewis, "Conformal printing of electrically small antennas on three-dimensional surfaces," Adv. Mater., vol. 23, pp. 1335-1340, 2011.

[19] C. Pfeiffer, X. Xu, S. R. Forrest, and A. Grbic, "Direct transfer patterning of electrically small antennas onto three-dimensionally contoured substrates," Adv. Mater., vol. 24, pp. 1166-1170, 2012.

[20] J. Wu, A. H. Abdelrahman, M. Liang, X. Yu, and H. Xin, "Monopole antenna radiation pattern control via 3D-printed dielectrics," IEEE Trans. Antennas Propag., vol. 65, no. 8, pp. 3869-3876, Aug. 2017.

[21] K.-L Wong, Compact and Broadband Microstrip Antennas, New York: Wiley, ch. 3, 2002.

[22] J.-Y. Pang, S.-Q. Xiao, S.-T. Chen, and B.-Z. Wang, "Compact and wideband PIFA for DCS/PCS/UMTS/WLAN communication system," Microw. Opt. Technol. Lett., vol. 52, pp. 1097-1100, May 2010.

[23] M.-C. Tang, T. Shi, X. Hong, and S. Qing, "Low-profile asymmetrical-CSRR-loaded stacked microstrip patch antenna," Appl. Comput. Electromagn. Soc. J., vol. 30, no. 8, pp. 850-854, Aug. 2015.

[24] M. Mirzaee and S. Noghanian, "Additive manufacturing of a compact 3D dipole antenna using ABS thermoplastic and high temperature carbon paste," in Proc. IEEE Int. Symp. Antennas Propag., pp. 475-476, Jun. 2016.

[25] R. W. Ziolkowski, P. Jin and C.-C. Lin, "Metamaterial-inspired engineering of antennas," Proc. IEEE, vol. 99, no. 10, pp. 1720-1731, Oct. 2011.

[26] F. V. D. Bergh, A. P. Engelbrecht, "A study of particle swarm optimization particle trajectories," Inform. Sci., vol. 176, no. 8, pp. 937-971, 2006

[27] K. Tang, Z. Li, L. Luo, and B. Liu, "Multi-strategy adaptive particle swarm optimization for numerical optimization," Eng. Appl. Artif. Intell., vol. 37, pp. 9-19, Jan. 2015.

[28] S. R. Best, "The radiation properties of electrically small folded spherical helix antennas," IEEE Trans. Antennas Propaga., vol. 52, no. 4, pp. 953-960, 2004. 